\title{
Incorporating ulipristal acetate in the care of symptomatic uterine fibroids: a Canadian cost- utility analysis of pharmacotherapy management
}

This article was published in the following Dove Press journal:

ClinicoEconomics and Outcomes Research

17 April 2015

Number of times this article has been viewed

\author{
Bernice Tsoi ${ }^{1,2}$ \\ Gord Blackhouse ${ }^{1,2}$ \\ Simon Ferrazzi ${ }^{3}$ \\ Clare J Reade ${ }^{4}$ \\ Innie Chen ${ }^{5,6}$ \\ Ron Goeree ${ }^{1,2,7}$
}

'Department of Clinical Epidemiology and Biostatistics, McMaster University, Hamilton, ON, Canada; ${ }^{2}$ Programs for Assessment of Technology in Health (PATH) Research Institute, St Joseph's Healthcare Hamilton, Hamilton, ON, Canada; ${ }^{3}$ ndependent Consultant, Oakville, ON, Canada; ${ }^{4}$ Division of Gynecologic Oncology, University of Toronto, Toronto, ON, Canada; ${ }^{5}$ Department of Obstetrics and Gynaecology, University of Ottawa, ${ }^{6}$ Ottawa Hospital Research Institute, Ottawa, ON, Canada; ${ }^{7}$ Centre for Evaluation of Medicines (CEM), St Joseph's Healthcare Hamilton, Hamilton, ON, Canada
Correspondence: Bernice Tsoi Programs for Assessment of Technology in Health (PATH) Research Institute, 25 Main Street West, Suite 2000, Hamilton, ON, Canada L8P IHI

Tel + I 9055237284

$\mathrm{Fax}+\mathrm{I} 9055220568$

Email tsoib@mcmaster.ca
Objective: To present a Canadian economic evaluation on the cost-utility of ulipristal acetate (5 mg orally daily) compared to leuprolide acetate $(3.75 \mathrm{mg}$ intramuscular monthly) in the treatment of moderate-to-severe symptoms of uterine fibroids in women eligible for surgery.

Methods: A probabilistic decision tree was constructed to model the pre-operative pharmacological management of uterine fibroids under the primary perspective of the Ontario public payer. The model parameterized data from clinical trials, observational studies, and public costing databases. The outcome measure was the incremental cost-utility ratio. Uncertainty in the model was explored through sensitivity and scenario analyses.

Results: Ulipristal was associated with faster control of excessive menstrual bleeding, fewer symptoms of hot flashes and lower health care resource consumption. The ulipristal strategy dominated leuprolide as it provided patients with more quality-adjusted life years $(0.177$ versus $0.165)$ at a lower cost $(\$ 1,273$ versus $\$ 1,366)$. Across a range of sensitivity analyses, the results remained robust except to the dose of the comparator drug. If leuprolide was administered at $11.25 \mathrm{mg}$, once every 3 months, the expected cost for the leuprolide strategy would decline and the associated incremental cost-utility ratio for ulipristal would be $\$ 168$ /quality-adjusted life year.

Conclusion: Ulipristal offers a unique opportunity to effectively and rapidly control menstrual bleeding in patients with uterine fibroids; thereby improving their quality of life while minimizing the probability of moderate-to-severe hot flashes that are common with leuprolide. The current economic analysis suggests that ulipristal remains the dominant strategy across extensive sensitivity analyses.

Keywords: cost-utility analysis, uterine leiomyomas, preoperative care, decision tree, menorrhagia

\section{Introduction}

Uterine leiomyomas (uterine fibroids) are benign, hormone-sensitive tumors within the uterine wall composed of smooth muscle cells and connective tissue. ${ }^{1,2}$ Although the majority of patients are asymptomatic, ${ }^{2}$ for the subset who are symptomatic, fibroids are associated with heavy menstrual bleeding, pelvic pain, and bulk-related complaints that often compromise a patient's quality of life..$^{3-6}$ The most common symptom reported among patients is menorrhagia which may lead to iron-deficient anemia and chronic fatigue. ${ }^{7}$

In the treatment of symptomatic uterine fibroids, current guidelines have recommended a surgical approach as it represents the only curative treatment available in a clinician's toolkit. ${ }^{8,9}$ Pre-operative management may however be desirable to optimize 
a patient's medical condition to reduce subsequent surgical morbidity, ${ }^{10,11}$ especially given the wait times associated with elective surgeries. Until recently, few pharmacological options have been available for symptom management as most patients would have already tried oral contraceptives, intra-uterine devices, nonsteroidal anti-inflammatory drugs and/or iron supplements during the course of their illness without successful symptom control. These agents have shown marginal benefit in managing bleeding and bulk symptoms associated with uterine fibroids. ${ }^{10}$ Instead, an alternative frequently prescribed by physicians in Canada is the off-label usage of a gonadotropin-releasing hormone (GnRH) agonist, such as leuprolide acetate, since it is considered to be the most effective medical therapy in controlling bleeding, correcting anemia, and reducing fibroid size. ${ }^{8}$ However, concerns remain with the use of GnRH agonists given their suboptimal side-effect profile (eg, flare effects, menopausal symptoms), especially in the long-term (eg, potential for bone mineral loss); their delayed onset of bleeding control; and the rapid nature of fibroid regrowth upon treatment cessation. ${ }^{1,2,12-14}$

A new option, ulipristal acetate, was approved in Canada in 2013 and is now part of the medical toolkit for uterine fibroid symptom management in women eligible for surgery. In a study comparing 3-month treatment of ulipristal to leuprolide, ulipristal demonstrated non-inferiority to oncemonthly leuprolide in controlling excessive uterine bleeding and was significantly less likely to cause symptoms of hot flashes. ${ }^{13}$ In addition, at 6 months follow-up after a single treatment course, patients on ulipristal maintained sustained fibroid reduction. ${ }^{13}$ The publication of this trial's results has left practitioners and decision-makers questioning the potential economic value of a practice change if ulipristal is prescribed. To date, there has been no Canadian economic evaluation comparing pharmacotherapies to manage symptoms of uterine fibroids. This paper thus investigates the economic value of ulipristal compared to leuprolide in the management of moderate-to-severe symptoms from uterine fibroids in patients for whom surgery is an option. Given the significant heterogeneity observed in clinical practice, we further conducted scenario analysis to explore the costutility of pharmacotherapy for uterine fibroids under different settings and perspectives.

\section{Materials and methods}

A probabilistic decision tree was used to estimate the expected costs and quality-adjusted life years (QALYs) of two medical treatments for symptomatic uterine fibroids: ulipristal (5 mg orally daily) compared to leuprolide (3.75 mg intramuscular monthly) (Figure 1). A decision tree analysis was adopted given the acute nature of pre-surgical drug therapy. ${ }^{15}$ The primary perspective of this model was that of the public health care payer (ie, Ontario's Ministry of Health and Long Term Care) with only direct medical costs related to the intervention and the condition captured. The model's time horizon captured a pre-surgical period of 3 months to reflect the licensed indication for ulipristal. ${ }^{16}$ The Consolidated Health Economic Evaluation Reporting Standards were followed in reporting this economic evaluation. ${ }^{17}$

\section{Health states and transitions probabilities}

The health states in this model were defined a priori according to menstrual bleeding control. All patients began with

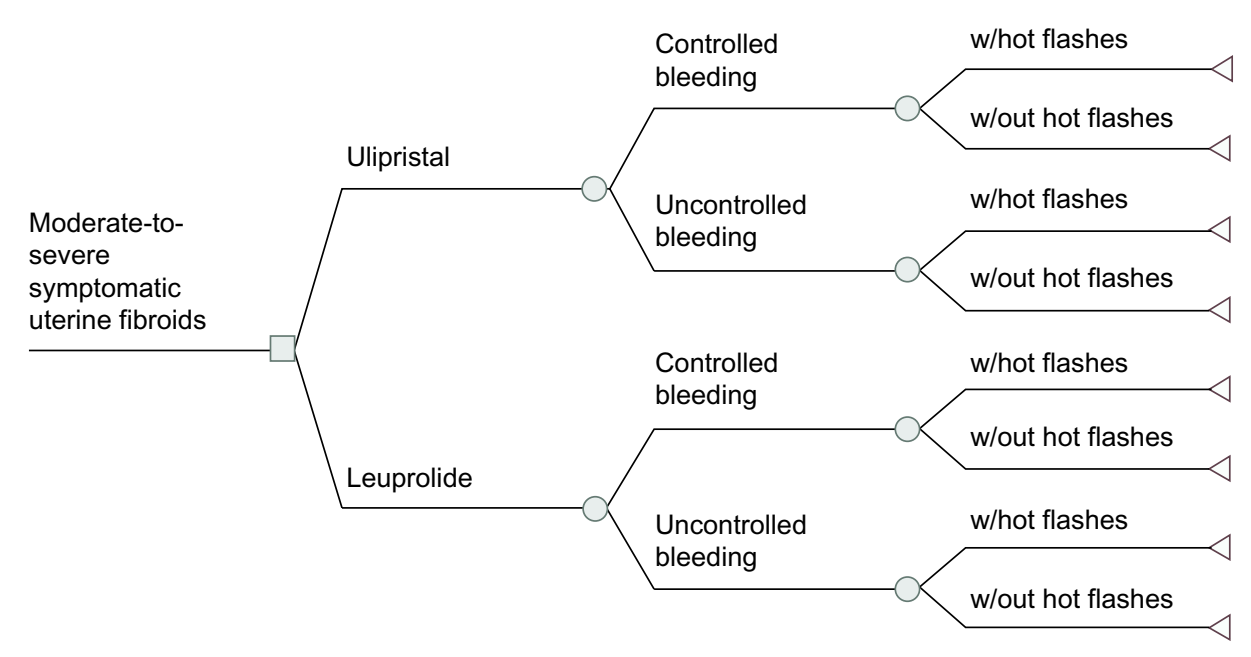

Figure I Structure of the economic model.

Note: This decision tree illustrates the structure of the model alongside the health states that were incorporated into this model. Abbreviation: w/, with. 
symptomatic uterine fibroids and, over the course of their treatment, either achieved "controlled bleeding" (ie, defined in alignment with the PGL4001 [UPA] Efficacy Assessment in Reduction of Symptoms due to Uterine Leiomyomata [PEARL] clinical trial, as a monthly pictorial blood assessment chart [PBAC] score $<75$, which corresponds to a blood loss $\leq 60 \mathrm{~mL} ;{ }^{18}$ or "uncontrolled bleeding" [PBAC score $\geq 75]$ ). ${ }^{13}$ The transition probabilities for these health states were taken from PEARL II, a head-to-head randomized-controlled trial that compared ulipristal against leuprolide. This study included premenopausal female patients, aged 18-50, with heavy uterine bleeding due to their fibroids (pre-treatment monthly PBAC $>100$, which corresponds to a blood loss of $80 \mathrm{~mL}$ ) and at least one myoma measuring between $3-10 \mathrm{~cm}$ in diameter. ${ }^{13}$ At 3 months of therapy, a PBAC score $<75$ was achieved by $90.3 \%$ and $89.1 \%$ of the patients treated with ulipristal and leuprolide respectively (Table 1$)^{13}$ (difference: $1.2 \%$ [95\% confidence interval: $-9.3 \%, 11.8 \%]$ ).

Among the proportion of patients who achieved bleeding control, the model also incorporated the time-to-controlled bleeding given the drug's differences in onset of action. ${ }^{13}$ Onset of action was defined as the first day in which the total PBAC score for the following 28 days and for all subsequent 28-day periods (calculated daily) until the end of the treatment, was less than $75 .^{13}$ The number of days of controlled menstrual bleeding was determined through KaplanMeier analysis of patient-level data from the PEARL II study as supplied by the study investigators (Table 1). As discussed within the appropriate sections, data on time-to-controlled bleeding were incorporated into the utilities calculation and impacted the number of lost productivity days when addressing the secondary societal perspective.

The probability of moderate-to-severe hot flashes, a drug-related adverse effect, was also incorporated into the model (Table 1). Experiencing hot flashes was not specific to whether patients responded to therapy.

\section{Utilities and QALYs}

Effectiveness in this model was translated into QALYs. Utility weights for each health state were derived from a crosssectional direct elicitation exercise of 909 pre-menopausal women across Canada (Table 1). ${ }^{19}$ Respondents were asked to rate clinically validated health states that described different symptom-levels of uterine fibroids on the EuroQol5dimensions-5 levels ${ }^{20}$ according to five dimensions: mobility, self-care, usual activities, pain/discomfort and anxiety/depression. This exercise further asked respondents to place a value on hot flashes and mode of drug administration (Table 1). ${ }^{19}$

\section{Costs and resource utilization}

Costs were taken from a variety of sources with all costs reported in 2013 Canadian dollars. As this model captures the pre-surgical period, costs were calculated from the initial presentation (ie, surgical consultation) until the completion of the drug regimen before undergoing surgery. The costs of the surgical work-up and subsequent surgery were not included in this analysis as this would apply to all patients, irrespective of their initial pre-surgical pharmacotherapy. Between the two pharmacotherapies, a difference in hospital-related surgical cost was not to be expected..$^{13}$ An $8 \%$ pharmacy markup was applied to all drug prices ${ }^{21}$ alongside a pharmacist's dispensing fee. ${ }^{22}$ The monthly cost of leuprolide $(3.75 \mathrm{mg}$ intramuscular) and of ulipristal (5 mg orally) was $\$ 347.18$ and $\$ 343.80$ respectively. ${ }^{23}$ No costs were associated with managing adverse drug reactions, such as hot flashes, as it was assumed that patients would be counseled on conservative measures to handle these.

Each leuprolide injection was assumed to lead to a billing for the drug administration and a physician's partial assessment (physician opinion). A chart audit conducted in Ontario suggested that $68 \%$ of injections were conducted at the specialist's office while the remainder were performed in a general practitioner setting (unpublished data from IMS Brogan [IMS Brogan is the Canadian business unit of IMS Health that provides anonymized medical claims data], 2013). A weighted injection cost was thus calculated according to these proportions. For ulipristal, patients were assumed to require one specialist's visit over the treatment course (Table 1).

No other costs were included in the base-case analysis beyond those mentioned above. The resource utilization mentioned here was not specific to a patient's bleeding status.

Costs and utilities were not discounted given that the model time horizon was less than a year.

\section{Data and uncertainty analysis}

The incremental cost-utility ratio (ICUR) was calculated according to conventional decision rules with no ICUR calculated under situations of dominance.

The probabilistic analysis, based on Monte Carlo simulation techniques, ran the model over a large number of times $(n=1,000)$ with parameter values randomly drawn according to their distribution ${ }^{15}$ to permit exploration of parameter uncertainty. The type of distribution for each parameter was selected 


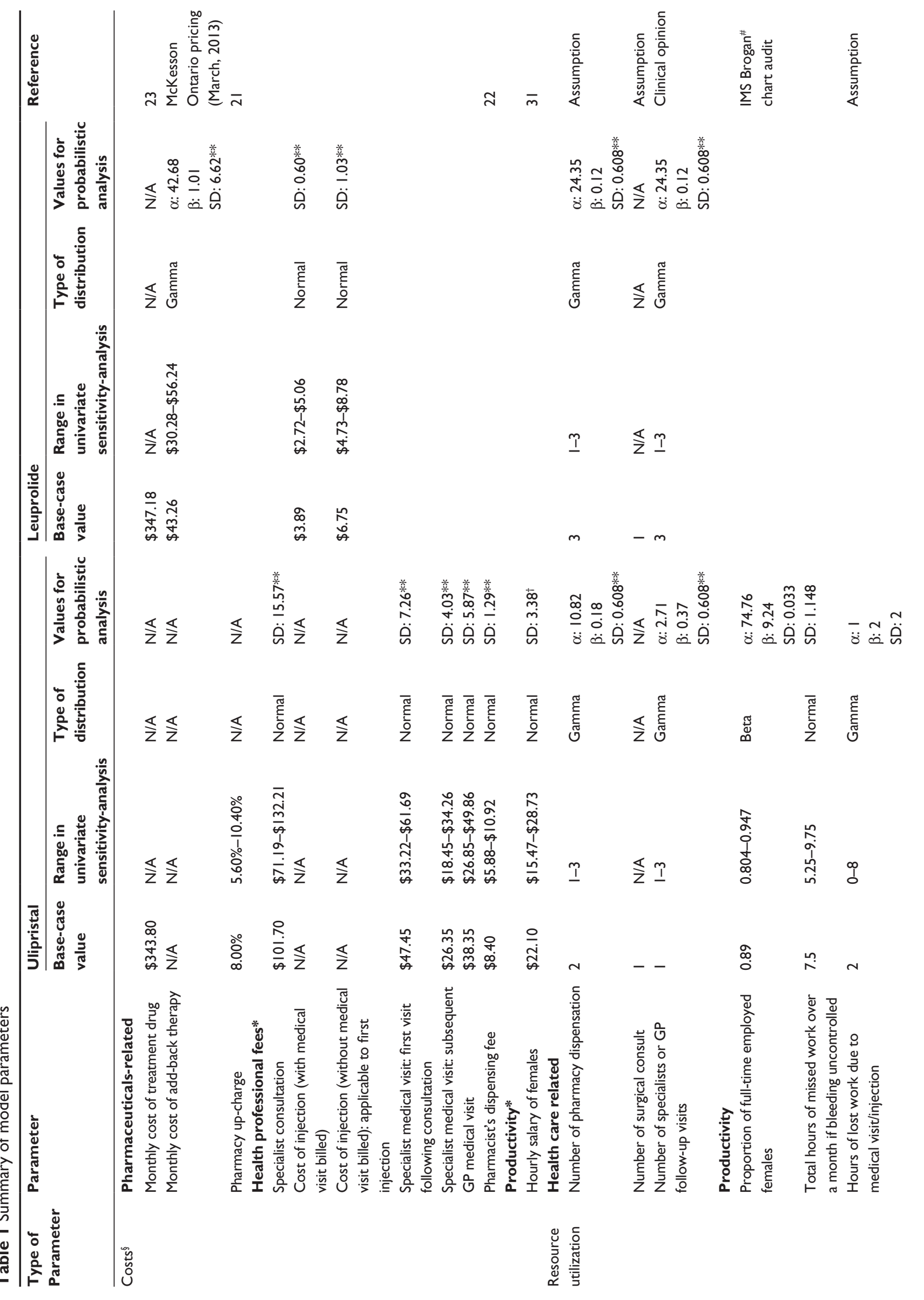


요

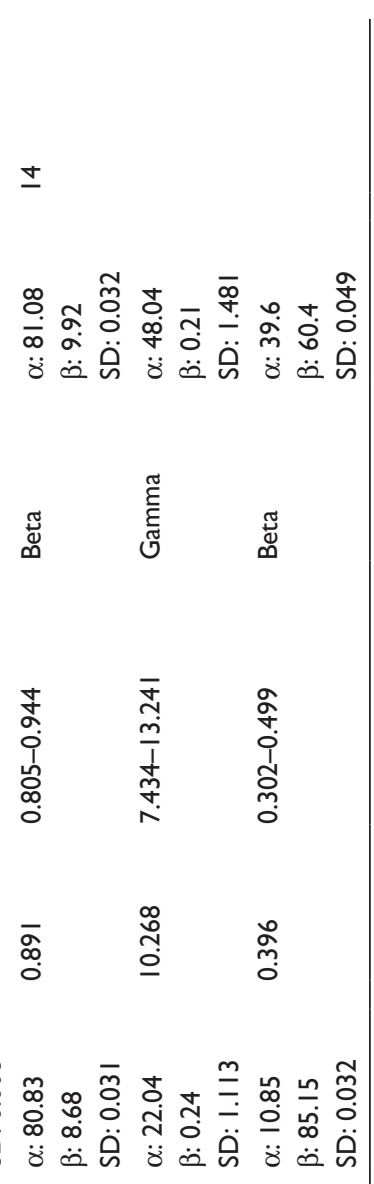

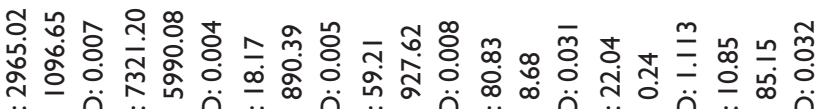

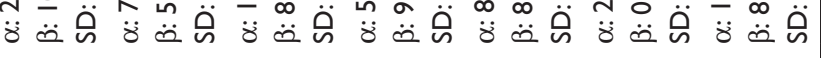

Ф๊ٓ

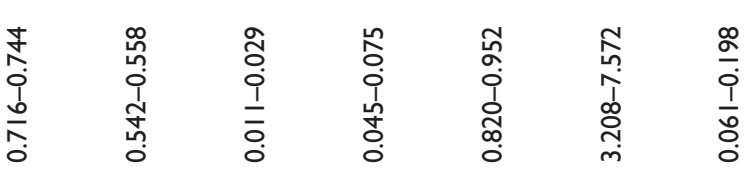

ก̂.
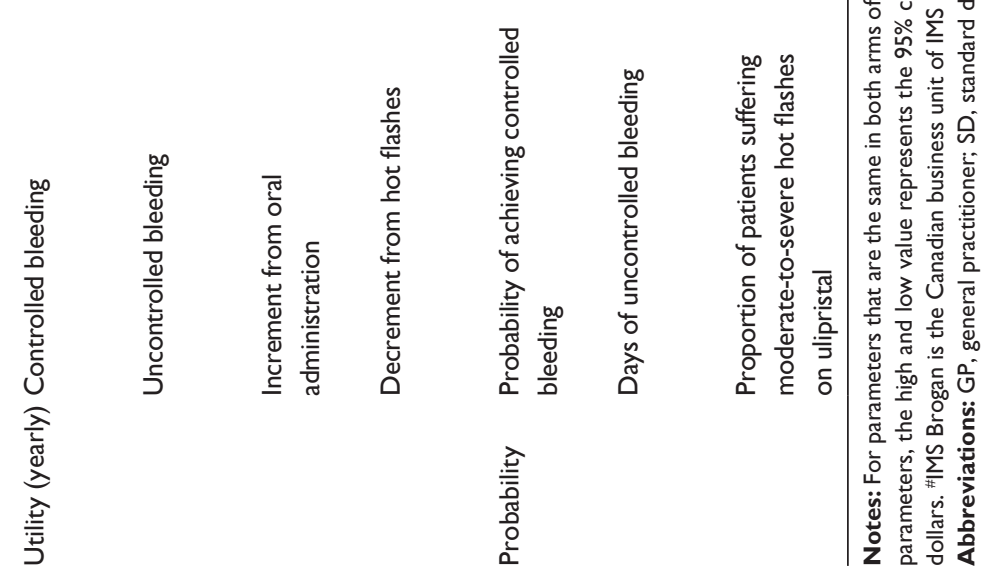
according to good modeling practice guidelines (eg, beta distribution for probabilities and utilities given that their values are constrained between 0 to 1 ; gamma distribution for resource utilization given the minimum asymptote of 0$).{ }^{24}$ Drug costs under the Ontario Drug Benefit plan were not defined by a probability distribution given that these are fixed costs across provinces. Rather, to reflect variability in drug costs, we modeled scenarios at different drug strengths. Parameter uncertainty based on the probabilistic analysis was presented by the cost-effectiveness acceptability curve (CEAC) which demonstrates the probability that a treatment (eg, ulipristal) is the most cost-effective compared to its alternative (eg, leuprolide) across a range of willingness-to-pay thresholds.

Methodological assumptions inherent to the model were also tested. We explored how the: i) removal of the utility increments or decrements associated with the mode of drug administration and side-effects respectively, or ii) removal of the billing of a medical visit alongside an injection visit for leuprolide impacted the model results. All model parameters, with the exception of the price of ulipristal and leuprolide, were further explored through one-way sensitivity analysis in which model inputs were tested individually to ascertain the impact of each parameter. ${ }^{15}$

The base-case scenario was populated with the parameters presented in Table 1. However, alternative scenarios were explored to ascertain the cost-utility of ulipristal under different situations, including:

1. Inclusion of add-back therapy: hormonal add-back may be prescribed to patients on leuprolide to manage adverse effects. A Canadian chart audit on physician practices suggested that $39 \%$ of uterine fibroid patients were given add-back therapy as part of their leuprolide regimen (IMS Brogan, unpublished data, 2013). Although the original model assumed no patients were receiving add-back therapy, two-way sensitivity analysis was conducted in which the proportion of patients receiving add-back therapy and the probability of hot flashes were changed simultaneously. The cost of add-back therapy included the price of $5 \mathrm{mg}$ norethindrone acetate, the pharmacy up-charge, and an additional dispensing claim.
2. Leuprolide $11.25 \mathrm{mg}$ : this single 3 -month dosing would reduce resource utilization in terms of specialists visits and injection-related costs.

3. Societal perspective: as fibroids primarily affect women of reproductive age, ${ }^{25,26}$ it can negatively impact productivity $^{27-30}$ and a societal perspective, incorporating the costs of work absenteeism, was undertaken. A chart review, conducted by IMS Brogan, suggests that approximately $89 \%$ of Canadian uterine fibroid patients are employed (unpublished data). It was therefore assumed that each medical visit would translate to 2 hours of lost productivity among employed females with the salary taken from published average wage rates of Canadian females. ${ }^{31}$ Furthermore, lost productivity due to uncontrolled bleeding was incorporated. In a Canadian survey of 331 employed females with moderate-to-severe uterine fibroids symptoms, patients reported that, over a month, the average number of missed work hours due to fibroidrelated symptoms was 7.5 hours. ${ }^{32}$

\section{Results}

Under the base-case, the expected costs for ulipristal and leuprolide were $\$ 1,273$ and $\$ 1,366$ while the expected QALYs were 0.177 and 0.165 respectively. Ulipristal was the dominant strategy in managing women suffering from moderate-to-severe symptoms of uterine fibroids as, on average, it led to a cost saving of $\$ 92$ and an incremental gain of 0.012 QALYs per patient over a 3-month period (Table 2). A breakdown of the different types of costs, by treatment strategy, is provided in Table 3. The distribution of all 1,000 Monte Carlo simulations lay in the south-east quadrant of the incremental cost-effectiveness plane (Figure 2). As such, no CEAC is presented as, across all willingness-to-pay thresholds, ulipristal had a $100 \%$ probability of being the most cost-effective strategy. Furthermore, the economic model was robust across all the parameters tested in the one-way sensitivity analysis (Table 4).

The results remained robust to the removal of utility increments/decrements with ulipristal being the dominant strategy (Table 5). Another conservative assumption tested

Table 2 Expected results under the base-case scenario

\begin{tabular}{lllllll}
\hline & Total & & & \multicolumn{1}{l}{ Incremental } & & \\
\cline { 2 - 3 } & Costs & QALYs & & Costs & QALYs & ICUR \\
\hline Leuprolide & $\$ 1,365.58$ & 0.165 & & -reference- & -reference- & \\
Ulipristal & $\$ 1,273.44$ & 0.177 & & $\$-92.13$ & 0.012 & Ulipristal dominates leuprolide \\
\hline
\end{tabular}

Note: Costs in 2013 Canadian dollars.

Abbreviations: QALYs, quality-adjusted life years; ICUR, incremental cost-utility ratio. 
Table 3 Expected cost breakdown under the base-case scenario (deterministic results)

\begin{tabular}{|c|c|c|c|}
\hline Type of costs & Ulipristal & Leuprolide & $\begin{array}{l}\text { Difference (ie, ulipristal - } \\
\text { leuprolide) }\end{array}$ \\
\hline \multicolumn{4}{|l|}{ Drug-related costs $(\$)$} \\
\hline Drug costs & $1, \mid 13.91$ & $\mathrm{I}, \mathrm{I} 24.86$ & -10.95 \\
\hline Pharmacist dispensation & 8.40 & 25.20 & -16.80 \\
\hline Drug administration & 0.00 & 113.44 & -113.44 \\
\hline Total drugs & $1, \mid 22.31$ & $1,263.50$ & $-14 \mid .19$ \\
\hline \multicolumn{4}{|l|}{ Non-drug costs } \\
\hline Medical visits* & 149.15 & 101.7 & 47.45 \\
\hline Total direct health care costs & I,27।.46 & $1,365.20$ & -93.74 \\
\hline Cost from time lost from work due to symptoms & 66.13 & 93.23 & -27.10 \\
\hline Cost from time lost from work due to medical visits & 78.68 & 157.35 & -78.67 \\
\hline Total indirect health care costs & $|44.8|$ & 250.58 & -105.77 \\
\hline
\end{tabular}

Notes: *Medical visits in which patients are assessed by the physician and receive an injection are not captured here. Rather, such costs have been classified under "drug administration". Costs in 2013 Canadian dollars.

was the impact of medical visits associated with each injection. If patients on leuprolide had no further billed medical visits except for their initial consult, the leuprolide strategy was found to be less costly $(\$ 1,263$ versus $\$ 1,274)$ (Table 5). As the QALYs remained unchanged (0.165 QALYs versus 0.177 QALYs), the ICUR for ulipristal became \$1,015.65/QALY and, as the willingness-to-pay threshold rose, the probability that ulipristal was costeffective increased (Figure 3).

Three alternate scenarios were tested and, with the exception of the dosage of leuprolide (scenario 2), ulipristal remained the dominant scenario. Compared to basecase, incorporating add-back therapy reduced the QALY difference between the two treatment strategies with little

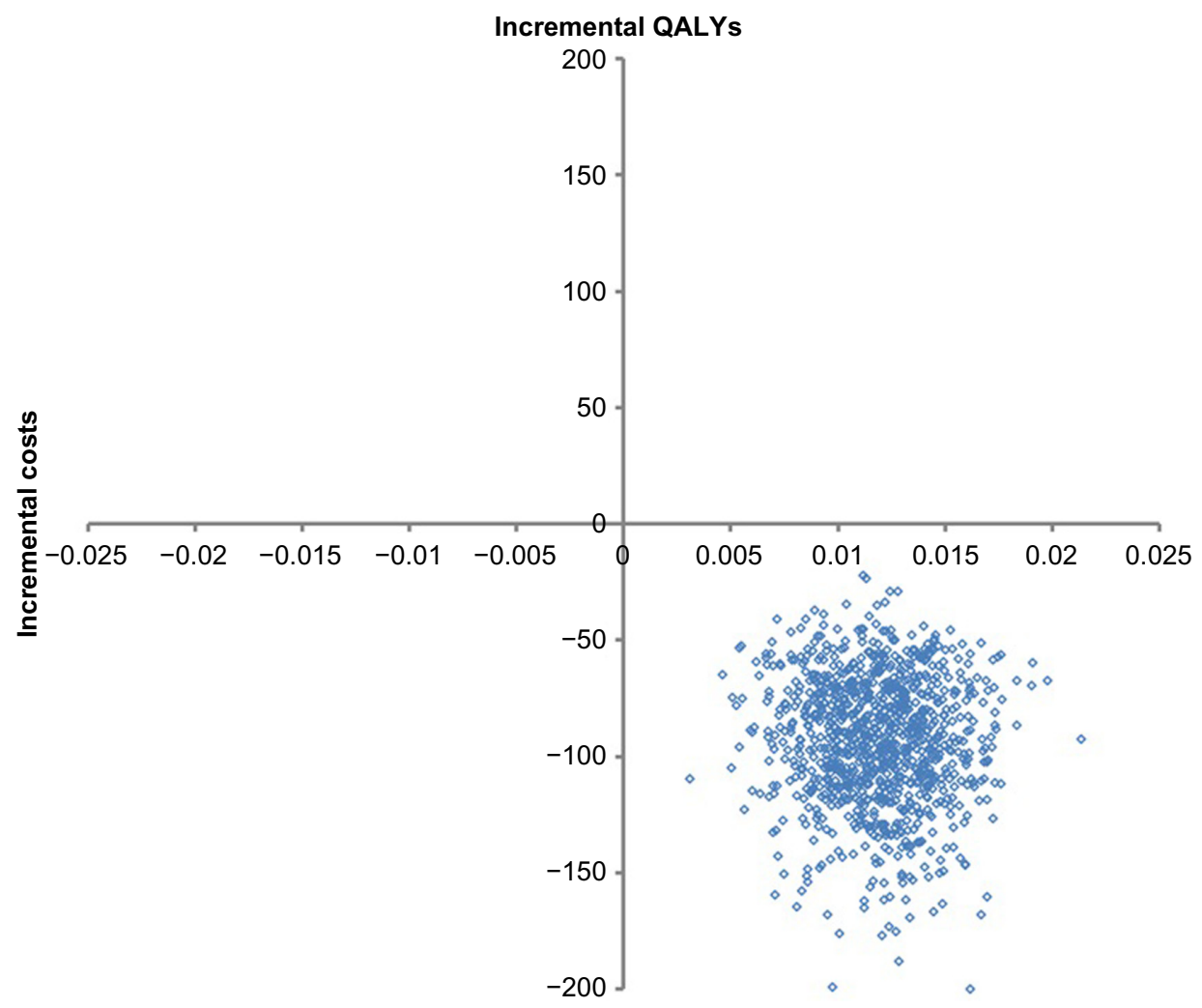

Figure 2 Probabilistic sensitivity analysis base-case (health care payer perspective).

Notes: The incremental cost-effectiveness plane demonstrates the joint distribution in costs (y-axis) and QALYs (x-axis), recalculated over I,000 replications of the study parameters using Monte Carlo simulation technique. Each point in the graph represents a single iteration of the Monte Carlo simulation.

Abbreviation: QALYs, quality-adjusted life years. 
Table 4 Results of one-way univariate sensitivity analysis of the base-case parameters

\begin{tabular}{|c|c|c|c|c|}
\hline \multirow[t]{2}{*}{ Parameter description } & \multicolumn{2}{|l|}{ Lower range } & \multicolumn{2}{|c|}{ Upper range } \\
\hline & $\begin{array}{l}\text { Parameter } \\
\text { value }\end{array}$ & $\begin{array}{l}\text { ICUR } \\
\text { result }\end{array}$ & $\begin{array}{l}\text { Parameter } \\
\text { value }\end{array}$ & $\begin{array}{l}\text { ICUR } \\
\text { result }\end{array}$ \\
\hline \multicolumn{5}{|l|}{ Base-case model parameters } \\
\hline Monthly cost of "add-back"-therapy, norethindrone acetate: $5 \mathrm{mg}$ & $\$ 30.28$ & Dominant & $\$ 56.24$ & Dominant \\
\hline Pharmacy upcharge & $5.60 \%$ & Dominant & $10.40 \%$ & Dominant \\
\hline ObGyn: consultation for initial diagnosis (20 minutes) & $\$ 71.19$ & Dominant & $\$ 132.21$ & Dominant \\
\hline Cost of injection - medical visit billed alongside & $\$ 2.72$ & Dominant & $\$ 5.06$ & Dominant \\
\hline Cost of first injection - sole reason (ie, no visit) & $\$ 4.73$ & Dominant & $\$ 8.78$ & Dominant \\
\hline ObGyn: specific assessment & $\$ 33.22$ & Dominant & $\$ 61.69$ & Dominant \\
\hline ObGyn: partial assessment & $\$ 18.45$ & Dominant & $\$ 34.26$ & Dominant \\
\hline GP: re-assessment during administration of $\mathrm{GnRH}$ & $\$ 26.85$ & Dominant & $\$ 49.86$ & Dominant \\
\hline Pharmacist's dispensing fee & $\$ 5.88$ & Dominant & $\$ 10.92$ & Dominant \\
\hline Number of times ulipristal dispensed & 1 & Dominant & 3 & Dominant \\
\hline Number of times leuprolide injected & 1 & Dominant & 3 & Dominant \\
\hline Number of specialists follow-up visits for ulipristal & 1 & Dominant & 3 & Dominant \\
\hline Proportion of females working full-time & 0.804 & Dominant & 0.947 & Dominant \\
\hline Proportion of hours lost to total hours worked if bleeding uncontrolled & 0.001 & Dominant & 0.127 & Dominant \\
\hline Proportion of patients with one absenteeism due to uncontrolled bleeding & 0.274 & Dominant & 0.515 & Dominant \\
\hline Hours worked per month & 168 & Dominant & 184 & Dominant \\
\hline Hours of lost work due to medical visit or injection & 0 & Dominant & 8 & Dominant \\
\hline Yearly utility of controlled bleeding & 0.716 & Dominant & 0.744 & Dominant \\
\hline Yearly utility uncontrolled bleeding & 0.542 & Dominant & 0.558 & Dominant \\
\hline Yearly utility increment for oral administration & 0.011 & Dominant & 0.029 & Dominant \\
\hline Yearly utility decrement from hot flashes & 0.045 & Dominant & 0.075 & Dominant \\
\hline Days of uncontrolled bleeding (leuprolide) & 7.434 & Dominant & $13.24 \mid$ & Dominant \\
\hline Days of uncontrolled bleeding (ulipristal) & 3.208 & Dominant & 7.572 & Dominant \\
\hline Proportion of patients suffering moderate-to-severe hot flashes (leuprolide) & 0.302 & Dominant & 0.499 & Dominant \\
\hline Proportion of patients suffering moderate-to-severe hot flashes (ulipristal) & 0.061 & Dominant & 0.198 & Dominant \\
\hline Probability of achieving controlled bleeding (leuprolide) & 0.805 & Dominant & 0.944 & Dominant \\
\hline Probability of achieving controlled bleeding (ulipristal) & 0.820 & Dominant & 0.952 & Dominant \\
\hline
\end{tabular}

Note: Costs in 2013 Canadian dollars.

Abbreviations: ICUR, incremental cost-utility ratio; ObGyn, obstetrics and gynecology; GnRH, gonadotropin-releasing hormone; GP, general practitioner.

impact on the costs (scenario 1) (Table 6). However, when the comparator was $11.25 \mathrm{mg}$ leuprolide (administered once every 3 months) (scenario 2), the expected cost for the leuprolide strategy reduced to $\$ 1,271$ (Table 6). The Monte Carlo simulations lay mainly in the northeast quadrant of the incremental cost-effectiveness plane (Figure 4A) with an ICUR of $\$ 168.39$ /QALY (Table 6). The CEAC suggested that, as the willingness-to-pay threshold increases, the probability in which ulipristal is the more cost-effective strategy, rises. At thresholds $\geq \$ 2,000$ / QALY, ulipristal was $100 \%$ the most likely cost-effective strategy (Figure 4B).

Under a societal perspective (scenario 3), ulipristal remained the dominant strategy (Table 6). Compared to the base-case scenario, the expected incremental QALY gained per patient remained relatively unchanged. Most of

Table 5 Expected results under different methodological assumptions

\begin{tabular}{|c|c|c|c|c|c|c|}
\hline & \multirow[t]{2}{*}{ Strategy } & \multicolumn{2}{|l|}{ Total } & \multicolumn{3}{|c|}{ Incremental } \\
\hline & & Costs & QALYs & Costs & QALYs & ICUR \\
\hline Utility increment of "oral & Leuprolide & $\$ 1,365.49$ & 0.165 & -reference- & -reference- & \\
\hline administration" removed & Ulipristal & $\$ 1,273.15$ & 0.172 & $\$-92.34$ & 0.007 & $\begin{array}{l}\text { Ulipristal dominates } \\
\text { leuprolide }\end{array}$ \\
\hline Utility decrement of & Leuprolide & $\$ 1,365.51$ & 0.171 & -reference- & -reference- & \\
\hline "hot flashes" removed & Ulipristal & $\$ 1,274.06$ & 0.178 & $\$-91.45$ & 0.008 & $\begin{array}{l}\text { Ulipristal dominates } \\
\text { leuprolide }\end{array}$ \\
\hline Medical visits not billed & Leuprolide & $\$ 1,262.77$ & 0.165 & -reference- & -reference- & \\
\hline during injection & Ulipristal & $\$ 1,274.24$ & 0.177 & $\$ 11.48$ & 0.012 & $\$ 1,015.65 / Q A L Y$ \\
\hline
\end{tabular}

Note: Costs in 2013 Canadian dollars.

Abbreviations: QALYs, quality-adjusted life years; ICUR, incremental cost-utility ratio. 


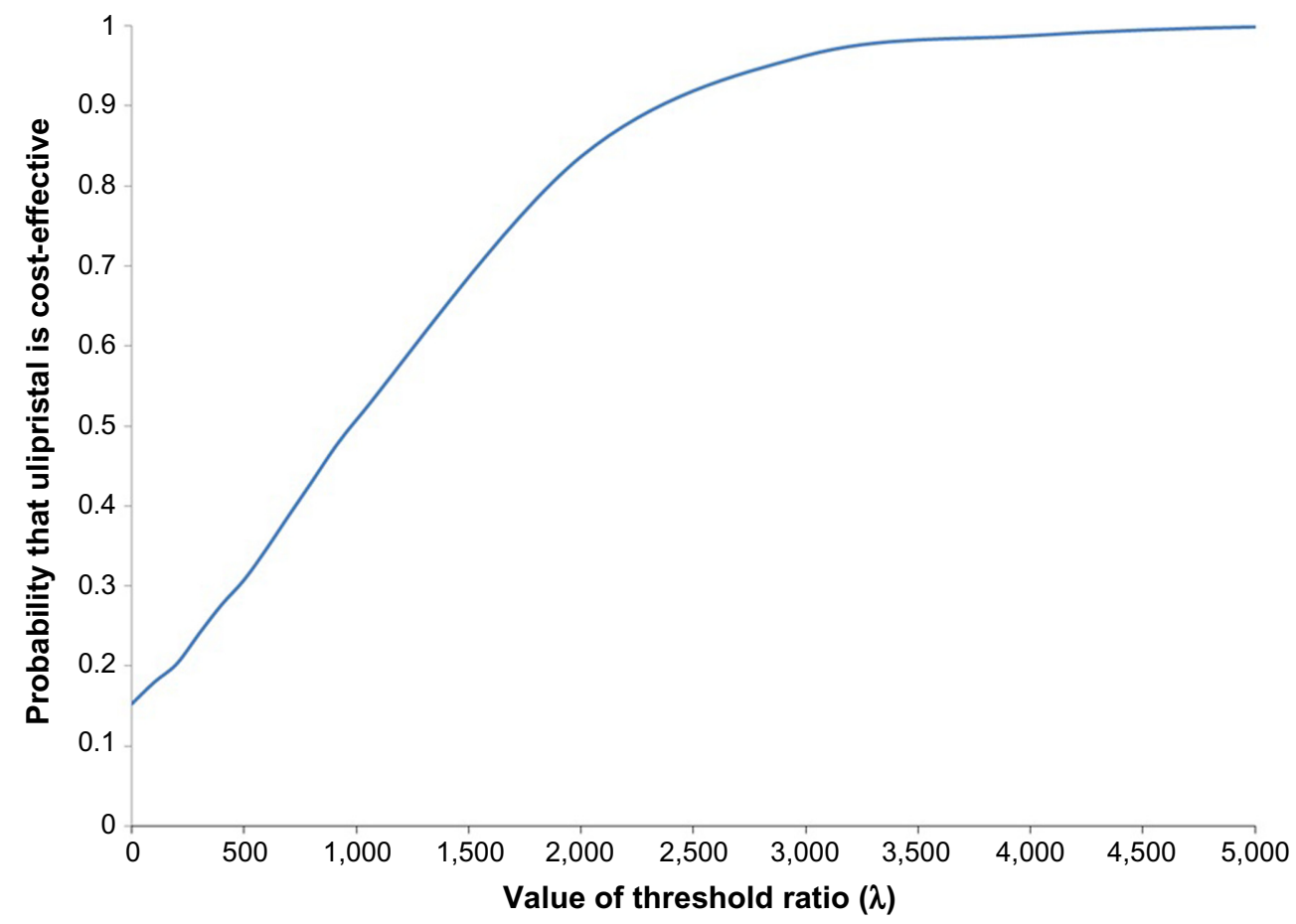

Figure 3 Cost-effectiveness acceptability curve (CEAC) - when medical assessments are not billed alongside each leuprolide injection.

Notes: The probabilistic sensitivity analysis is based on I,000 Monte Carlo simulations. The CEAC shows the likelihood to which ulipristal is cost-effective compared to leuprolide across a range of cost-effectiveness threshold values (x-axis). For each cost-effectiveness threshold (increasing from left to right) on the $\mathrm{x}$-axis, the proportion of iterations having ICUR at or below that threshold is plotted on the $y$-axis.

Abbreviation: ICUR, incremental cost-utility ratio.

1,000 Monte Carlo simulations ( $\mathrm{n}=999 ; 99.9 \%)$ lay in the south-east quadrant (Figure 5) under the societal perspective, indicative of little parameter uncertainty and, across all willingness-to-pay thresholds, ulipristal had a high probability (ie, $\geq 99.9 \%$ ) of being the most cost-effective strategy (CEAC not presented).

\section{Discussion}

Uterine fibroids can have a significant economic impact on the health care system and on society overall. Costof-illness studies have estimated that women with uterine fibroids have consistently higher use of medical services and procedure ${ }^{29}$ and that the cost of absenteeism and disability

Table 6 Expected results under different scenarios analyses

\begin{tabular}{|c|c|c|c|c|c|}
\hline \multirow[t]{2}{*}{ Scenario } & \multicolumn{2}{|l|}{ Total } & \multicolumn{3}{|c|}{ Incremental } \\
\hline & Costs & QALYs & Costs & QALYs & ICUR \\
\hline \multicolumn{6}{|c|}{ I. Inclusion of add-back therapy } \\
\hline \multicolumn{6}{|c|}{ Scenario a: $100 \%$ on add-back, no hot flashes in the leuprolide arm } \\
\hline Leuprolide & $\$ 1,366.36$ & $0.17 \mid$ & -reference- & -reference- & \\
\hline Ulipristal & $\$ 1,273.47$ & 0.177 & $\$-92.89$ & 0.006 & Ulipristal dominates leuprolide \\
\hline \multicolumn{6}{|c|}{ Scenario b: $39 \%$ on add-back, no hot flashes in the leuprolide arm } \\
\hline Leuprolide & $\$ 1,365.00$ & 0.171 & -reference- & -reference- & \\
\hline Ulipristal & $\$ 1,273.66$ & 0.177 & $\$-91.34$ & 0.006 & Ulipristal dominates leuprolide \\
\hline \multicolumn{6}{|c|}{ Scenario c: $39 \%$ on add-back, $50 \%$ reduction in hot flashes in the leuprolide arm } \\
\hline Leuprolide & $\$ 1,366.24$ & 0.168 & -reference- & -reference- & \\
\hline Ulipristal & $\$ 1,273.11$ & 0.177 & $\$-93.14$ & 0.009 & Ulipristal dominates leuprolide \\
\hline \multicolumn{6}{|c|}{ 2. I I.25 mg leuprolide } \\
\hline Leuprolide & $\$ 1,27 I .14$ & 0.165 & -reference- & -reference- & \\
\hline Ulipristal & $\$ 1,273.08$ & 0.177 & 1.93 & 0.012 & 168.39 \\
\hline \multicolumn{6}{|c|}{ 3. Societal perspective } \\
\hline Leuprolide & $\$ 1,614.50$ & 0.165 & -reference- & -reference- & \\
\hline Ulipristal & $\$ 1,4 \mid 6.77$ & 0.177 & $\$-197.74$ & 0.012 & Ulipristal dominates leuprolide \\
\hline
\end{tabular}

Note: Costs in 2013 Canadian dollars.

Abbreviations: QALYs, quality-adjusted life years; ICUR, incremental cost-utility ratio. 

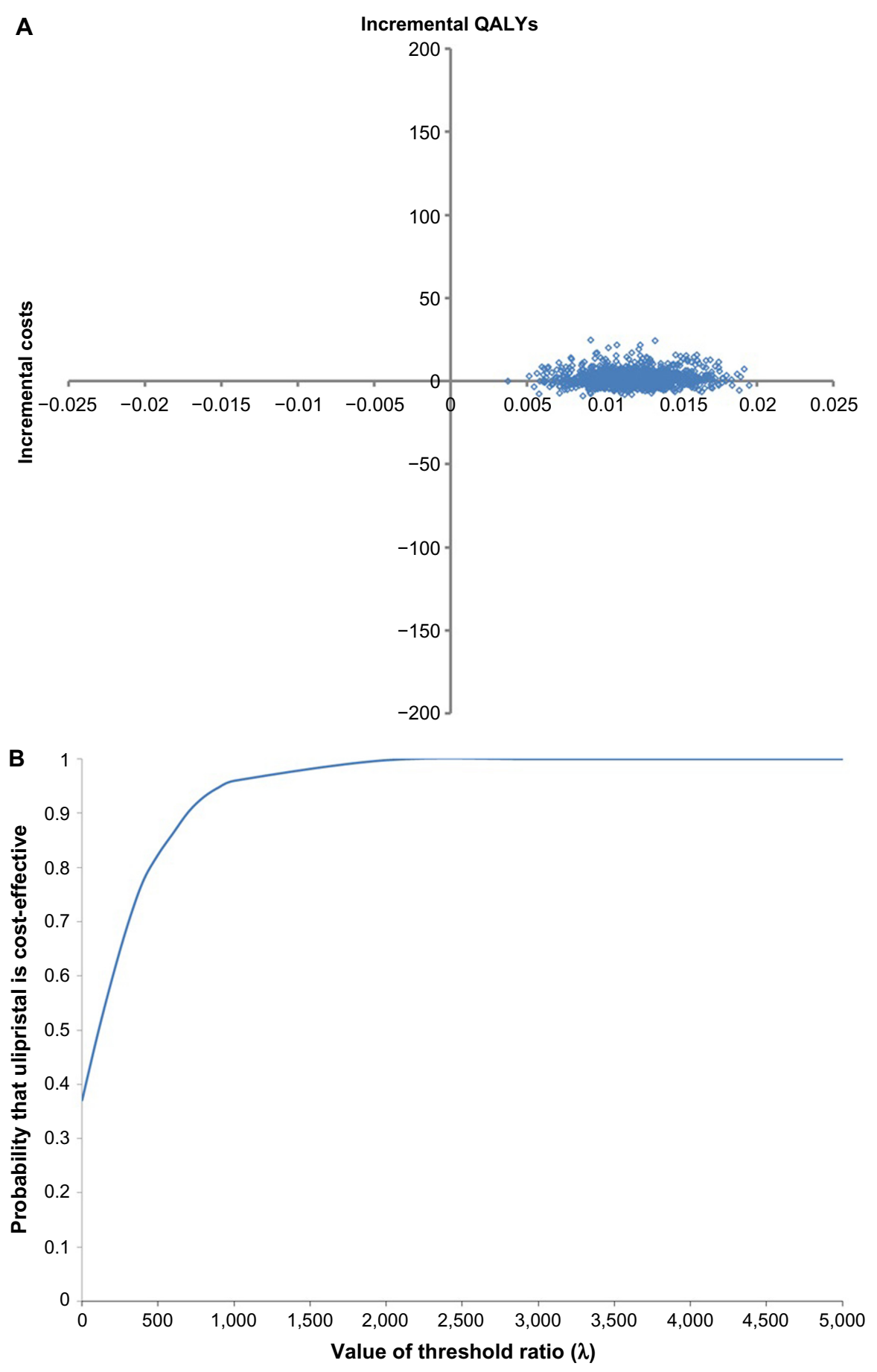

Figure 4 Probabilistic results when comparator is $11.25 \mathrm{mg}$, 3-month leuprolide (intramuscular).

Notes: (A) Probabilistic sensitivity analysis. (B) Cost-effectiveness acceptability curve. The probabilistic sensitivity analysis is based on I,000 Monte Carlo simulations. Abbreviation: QALYs, quality-adjusted life years.

is higher among women with uterine fibroids compared to controls. ${ }^{29,30,32}$ The management and treatment of uterine fibroids thus imposes a burden on the existing health care system and it is important to understand the economic implications between treatment options and the key cost drivers.
The introduction of ulipristal has raised numerous questions that can be addressed through an economic evaluation. Our analysis reveals that, across a wide range of scenarios, ulipristal is not only cost-effective but provides cost savings under both a Ministry of Health and a broader societal perspective within Canada for its current marketed indication. 


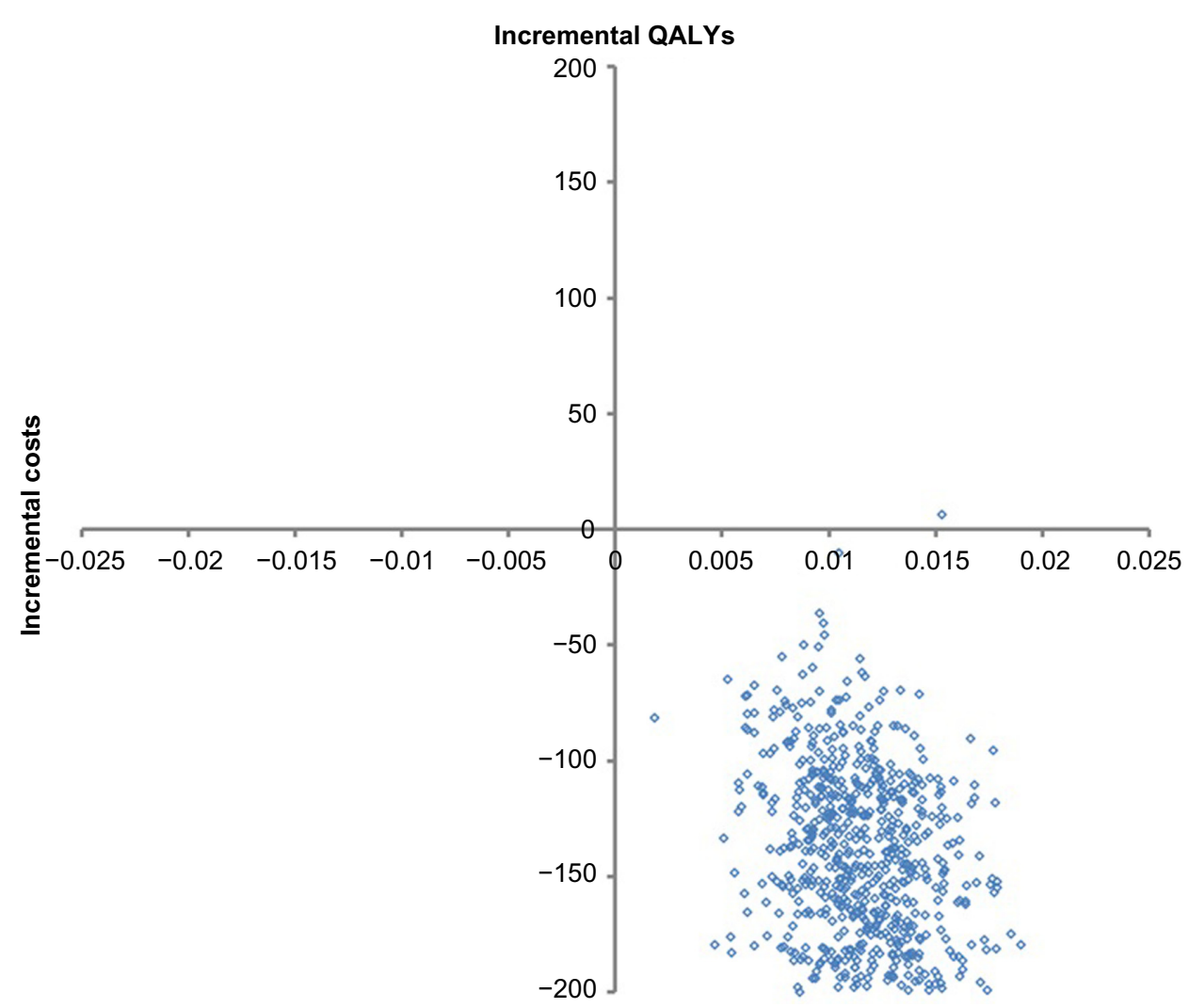

Figure 5 Probabilistic sensitivity analysis based on I,000 Monte Carlo simulations - under a societal perspective. Abbreviation: QALYs, quality-adjusted life years.

The model incorporated differences in projected medical visits, hot flashes and onset of action between ulipristal and leuprolide, as observed in PEARL II. Despite relatively small differences in QALYs and costs between the two treatment strategies, these differences were significant and, under a wide range of assumptions and sensitivity analyses, the model results remained robust. The model was most sensitive to the method of administration of leuprolide. When either no medical visit was billed alongside the injection or when leuprolide was administered as a single 3-month $11.25 \mathrm{mg}$ dose, ulipristal was no longer a dominant strategy. Despite this, the ICUR remained below the commonly quoted Canadian willingness-to-pay threshold of $\$ 20,000 / \mathrm{QALY}^{33}$ and, in incorporating stochastic uncertainty, ulipristal had $100 \%$ probability of being the most cost-effective strategy at this threshold level (Figure 3 and 5). It is important to note that this model has remained faithful to its current Health Canada indication and has found that 3-month treatment of ulipristal is cost-saving compared against leuprolide. Other indications have yet to be explored and we caution extrapolating the model results to other indications.

Recently, an economic evaluation has been published on ulipristal from a Hungarian perspective. ${ }^{34}$ In determining whether those results can be generalized to other settings, such as Canada, one must consider the International Society for Pharmacoeconomics and Outcomes Research guidelines on the transferability of health economic models across jurisdictions..$^{35}$ In these guidelines, models are suggested to be transferable unless significant variations exist either in the disease progression or in the clinical practice between jurisdictions. The published model reflected the clinical practice patterns in Hungary and compared ulipristal against: i) pre-surgical observation without medical management and ii) immediate hysterectomy. Neither of these comparators are appropriate within Canada or most developed countries, as hysterectomy is rarely an emergent procedure and leuprolide is often available as an adjunct prior to elective surgery. Our model thus addresses a different comparator to reflect the practices specific to Canada.

There are several limitations to this economic model. Firstly, adherence was not incorporated since discontinuation due to adverse events was found to be very low and nonsignificant within the existing trials. ${ }^{13,36}$ In PEARL II, discontinuation rates due to adverse events were $1 \%$ and $6 \%$ for ulipristal and leuprolide respectively. ${ }^{13}$ Discontinuation would mean that patients remain in a health state of "uncontrolled bleeding". As uncontrolled bleeding is associated with higher 
costs and lower utilities, if discontinuations were incorporated into this model, this would translate to a more favorable argument in support of ulipristal given its higher adherence rates. However, one of the model's simplifying assumptions was perfect compliance and this is expected to have led to more conservative findings. Secondly, it is important to recognize the limits of decision-analytic model in simulating reality. For instance, there exists considerable variation in terms of add-back regimens prescribed. The base-case model assumed no patients on add-back therapy and this, in itself, is a simplifying assumption. We therefore explored the impact of add-back regimen through scenario analysis and it was found to have little impact on the overall model's findings (scenario 1). Thirdly, some may consider a clinical outcome, such as "fibroid volume reduction", to be of greater interest as the effect measure in this economic analysis. The argument is that leuprolide is not only given for symptomatic relief but also to shrink the fibroid such that less invasive surgical procedures can become an option. ${ }^{11}$ However, the clinical trial comparing these two regimens in women with uterine fibroids found both medications reduced fibroids with no in-between statistical difference. Furthermore, the patient's subsequent type and route of surgery were similar following treatment on either drugs. ${ }^{13}$ Utility measure, as selected for this analysis, is a patient-focused outcome that captures an individual's preferences for a particular health condition. ${ }^{37}$ This effect measure is multi-dimensional (ie, pain, physical, social, and emotional) and is capable of capturing the broader impacts from a multitude of symptoms relating to uterine fibroids (eg, bulk, pain, and bleeding) alongside the adverse effects of pharmacotherapy. Utility further aligns with the existing Society of Obstetricians and Gynaecologists of Canada guidelines where a main treatment goal for uterine fibroids is improvement in quality of life. ${ }^{8}$ By combining utility weights with time (eg, time-to-bleeding control) to calculate QALYs, this measure of effect is far more sensitive and accurate in reflecting the pre-surgical differences between patient's outcome while on either of these two treatments. Lastly, decision-analytic models are constructed based on the available data and, in this case, certain model assumptions were based on unpublished observations. There are concerns that this may limit the model's validity and reproducibility. To address the first concern, we conducted extensive sensitivity analysis (ie, one-way sensitivity analysis, probabilistic analysis) to explore the uncertainty in these parameters. Given that the results remained robust across a range of sensitivity analyses, this provides increased confidence in the model results. For the latter concern on reproducibility, we have remained thoroughly transparent with the reporting of the model structure and its parameters.

In conclusion, our model brings to light the potential for dramatic impact on the existing management of patients with symptomatic uterine fibroids. By incorporating the clinical trial data alongside the resource utilization patterns and its associated treatment-related costs in Canada, this economic analysis suggests that ulipristal is cost-effective, if not cost-saving, under various scenarios tested from both health care payer and societal perspectives. Access to this new option should be made available to patients suffering from moderate-to-severe symptoms of uterine fibroids given its potential to improve a patient's quality of life while reducing health care expenditures compared to the current standard, leuprolide.

\section{Acknowledgments}

The authors thank Dr Wilfred Marvin Steinberg (St Michaels Hospital, Toronto, ON) and Dr Amanda Selk (Mount Sinai Hospital, Toronto, ON) for their assistance in providing their clinical expertise in validating this economic model.

\section{Disclosure}

This work was supported by a grant from the Mitacs Accelerate Internship program awarded to Bernice Tsoi. Matched funding was provided by both Mitacs and Actavis Specialty Pharmaceuticals Co. The authors of this publication had full and independent control over the methods, analyses and preparation of the final report and the economic model.

Bernice Tsoi is further supported through an Award from the Father Sean O'Sullivan Research Centre, St Joseph's Healthcare Hamilton. All authors report no other conflicts of interest in this work.

\section{References}

1. Murase E, Siegelman ES, Outwater EK, Perez-Jaffe LA, Tureck RW. Uterine leiomyomas: histopathologic features, MR imaging findings, differential diagnosis, and treatment. Radiographics. 1999;19(5): 1179-1197.

2. Wallach EE, Vlahos NF. Uterine myomas: an overview of development, clinical features, and management. Obstet Gynecol. 2004;104(2): 393-406.

3. Bukulmez O, Doody KJ. Clinical features of myomas. Obstet Gynecol Clin North Am. 2006;33(1):69-84.

4. Okolo S. Incidence, aetiology and epidemiology of uterine fibroids. Best Pract Res Clin Obstet Gynaecol. 2008;22(4):571-588.

5. Wegienka G, Baird DD, Hertz-Picciotto I, et al. Self-reported heavy bleeding associated with uterine leiomyomata. Obstet Gynecol. 2003; 101(3):431-437.

6. Zimmermann A, Bernuit D, Gerlinger C, Schaefers M, Geppert K. Prevalence, symptoms and management of uterine fibroids: an international internet-based survey of 21,746 women. BMC Womens Health. 2012;12:6. 
7. Segars JH, Parrott EC, Nagel JD, et al. Proceedings from the Third National Institutes of Health International Congress on Advances in Uterine Leiomyoma Research: comprehensive review, conference summary and future recommendations. Hum Reprod Update. 2014;20(3): 309-333.

8. Lefebvre G, Vilos G, Allaire C, et al. The management of uterine leiomyomas. J Obstet Gynaecol Can. 2003;25(5):396-418.

9. NHS National Institute for Health and Clinical Excellence. NICE Clinical Guideline 44: Heavy Menstrual Bleeding. London, UK: National Institute for Health and Clinical Excellence; 2007. Available from: http://www.nice.org.uk/guidance/cg44/resources/guidanceheavy-menstrual-bleeding-pdf. Accessed April 23, 2013.

10. Chabbert-Buffet N, Esber N, Bouchard P. Fibroid growth and medical options for treatment. Fertil Steril. 2014;102(3):630-639.

11. Lethaby A, Vollenhoven B, Sowter M. Efficacy of pre-operative gonadotrophin hormone releasing analogues for women with uterine fibroids undergoing hysterectomy or myomectomy: a systematic review. BJOG. 2002;109(10):1097-1108.

12. Batzer FR. GnRH analogs: options for endometriosis-associated pain treatment. J Minim Invasive Gynecol. 2006;13(6):539-545.

13. Donnez J, Tomaszewski J, Vazquez F, et al. Ulipristal acetate versus leuprolide acetate for uterine fibroids. $N$ Engl J Med. 2012;366(5): 421-432.

14. Evans P, Brunsell S. Uterine fibroid tumors: diagnosis and treatment. Am Fam Physician. 2007;75(10):1503-1508.

15. Briggs A, Claxton K, Sculpher M. Decision Modelling for Health Economic Evaluation. New York, NY: Oxford University Press; 2006.

16. Actavis Canada. Fibristal Product Monograph. Oakville, ON Watson Laboratories; 2013. Available from: http://www.actavis ca/NR/rdonlyres/04E31D66-291E-4603-92AE-0933A77D9B64/0/ Fibristal_Product_Monograph_English.pdf. Accessed February 8, 2015 .

17. Husereau D, Drummond M, Petrou S, et al. Consolidated Health Economic Evaluation Reporting Standards (CHEERS) statement. Int J Technol Assess Health Care. 2013;29(2):117-122.

18. Istre $\mathrm{O}$, Trolle B. Treatment of menorrhagia with the levonorgestrel intrauterine system versus endometrial resection. Fertil Steril. 2001;76(2):304-309.

19. Hux M, Ng C, Lozano-Ortega G, Ferrazzi S, Goeree R. Utility values for premenopasal women suffering from symptomatic uterine fibroids Expert Rev Pharmacoecon Outcomes Res. 2015;15(1):181-189.

20. Herdman M, Gudex C, Lloyd A, et al. Development and preliminary testing of the new five-level version of EQ-5D (EQ-5D-5L). Qual Life Res. 2011;20(10):1727-1736.

21. Patented Medicine Prices Review Board [homepage on the Internet]. Wholesale up-charge policies of Canada's public drug plan. Appendix C: Drug Plan Summaries; 2012. Available from: http://www.pmprb-cepmb. gc.ca/english/view.asp? $x=1591 \&$ mid=1495. Accessed February 8, 2015 .

22. Ontario Ministry of Health and Long-term Care [homepage on the Internet.] Ontario Drug Benefit Program: Dispensing fees; 2012. Available from: http://www.health.gov.on.ca/en/public/programs/drugs/programs/odb/opdp_dispensing_fees.aspx. Accessed February 8, 2015.
23. Ontario Ministry of Health and Long-term Care [homepage on the on the Internet]. Drugs funded by Ontario Drug Benefit (ODB) Program e-Formulary; 2013. Available from: http://www.health.gov.on.ca/en/pro/ programs/drugs/odbf_eformulary.aspx. Accessed February 8, 2015.

24. Briggs AH, Goeree R, Blackhouse G, O’Brien BJ. Probabilistic analysis of cost-effectiveness models: choosing between treatment strategies for gastroesophageal reflux disease. Med Decis Making. 2002;22(4): 290-308.

25. Rosati P, Exacoustos C, Mancuso S. Longitudinal evaluation of uterine myoma growth during pregnancy. A sonographic study. J Ultrasound Med. 1992;11(10):511-515.

26. Viswanathan M, Hartmann K, McKoy N, et al. Management of uterine fibroids: an update of the evidence. Evid Rep Technol Assess (Full Rep). 2007;(154):1-122.

27. Cote I, Jacobs P, Cumming D. Work loss associated with increased menstrual loss in the United States. Obstet Gynecol. 2002;100(4): 683-687.

28. Downes E, Sikirica V, Gilabert-Estelles J, et al. The burden of uterine fibroids in five European countries. Eur J Obstet Gynecol Reprod Biol. 2010;152(1):96-102.

29. Hartmann KE, Birnbaum H, Ben-Hamadi R, et al. Annual costs associated with diagnosis of uterine leiomyomata. Obstet Gynecol. 2006;108(4):930-937.

30. Lee DW, Ozminkowski RJ, Carls GS, et al. The direct and indirect cost burden of clinically significant and symptomatic uterine fibroids. $J$ Occup Environ Med. 2007;49(5):493-506.

31. Statistics Canada [homepage on the Internet]. Average hourly wages of employees by selected characteristics and profession, unadjusted data, by province (monthly) (Canada); 2013. Available from: http:// www.statcan.gc.ca/tables-tableaux/sum-som/101/cst01/labr69a-eng. htm. Accessed May 4, 2013.

32. Pron G, Cohen M, Soucie J, et al. The Ontario Uterine Fibroid Embolization Trial. Part 1. Baseline patient characteristics, fibroid burden, and impact on life. Fertil Steril. 2003;79(1):112-119.

33. Laupacis A, Feeny D, Detsky AS, Tugwell PX. How attractive does a new technology have to be to warrant adoption and utilization? Tentative guidelines for using clinical and economic evaluations. CMAJ. 1992;146(4):473-481.

34. Nagy B, Timar G, Jozwiak-Hagymasy J, et al. The cost-effectiveness of ulipristal acetate tablets in treating patients with moderate to severe symptoms of uterine fibroids. Eur J Obstet Gynecol Reprod Biol. 2014;175:75-81

35. Drummond M, Barbieri M, Cook J, et al. Transferability of economic evaluations across jurisdictions: ISPOR Good Research Practices Task Force report. Value Health. 2009;12(4):409-418.

36. Donnez J, Tatarchuk TF, Bouchard P, et al. Ulipristal acetate versus placebo for fibroid treatment before surgery. NEngl J Med. 2012;366(5): 409-420.

37. Whitehead SJ, Ali S. Health outcomes in economic evaluation: the QALY and utilities. Br Med Bull. 2010;96:5-21.
ClinicoEconomics and Outcomes Research

\section{Publish your work in this journal}

ClinicoEconomics \& Outcomes Research is an international, peerreviewed open-access journal focusing on Health Technology Assessment, Pharmacoeconomics and Outcomes Research in the areas of diagnosis, medical devices, and clinical, surgical and pharmacologica intervention. The economic impact of health policy and health systems

\section{Dovepress}

organization also constitute important areas of coverage. The manuscript management system is completely online and includes a very quick and fair peer-review system, which is all easy to use. Visit http://www.dovepress.com/testimonials.php to read real quotes from published authors. 